\title{
Core/Sheath Structured PVA Micro/Nano Composite Fibers for Biomedical Applications
}

\author{
Hong-Fei CHEN ${ }^{1, a}$, Dian-Luan QIU ${ }^{1, b}$, Li-Xing DAI ${ }^{1, c, *}$ \\ ${ }^{1}$ College of Chemistry, Chemical Engineering and Materials Science, Soochow \\ University, Suzhou, Jiangsu, 215123, People's Republic of China. \\ ahelloff_7086@163.com, 'billanqiu@163.com, dailixing@suda.edu.cn \\ ${ }^{*}$ Corresponding author
}

Keywords: Electrospinning, Composite Fibers, Core/Sheath, Antimicrobial Activity, Cell Adhesion.

\begin{abstract}
Modified electrospinning apparatus has been developed for continually fabricating nanofiber-coated microfibers. Two positively charged spinnerets were introduced and the PVA microfibers containing silver nanowires (AgNWs) were acted as the grounded collector. The prepared PVA/AgNWs microfiber moved through the electric field during electrospinning process and the nanofibers deposited onto the surface of the PVA/AgNWs microfiber to form a coating layer. Due to the addition of AgNWs, the micro/nano composite fibers had antimicrobial activity against Escherichia coli (ATCC 8099) and Staphylococcus aureus (ATCC 6538). In addition, the human osteoblasts attached well on the micro/nano composite fibers in a 3D direction.
\end{abstract}

\section{Introduction}

Poly(vinyl alcohol) (PVA), as one of the candidate materials for biomedical applications, has been widely used in the field of biology due to their nontoxicity, water-solubility, biodegradability and biocompatibility $[1,2]$. With the advantage of easy fiber-forming process, PVA is usually applied to prepare fibers. Recently, much attention has been paid to electrospinning as a unique technique, because it can produce polymer nanofibers with diameter in the range from nano- to micro-scale. The nanofibrous nonwoven mats produced by electrospinning have large surface area-to-volume ratio and high porosity with very small pore size. Therefore, they are particularly interesting for the field of biomedical engineering such as scaffolds for tissue engineering, drug delivery and wound dressings [3-5].

As biomaterials, nanofibrous nonwoven mats prepared by electrospinning are not satisfactory for fabricating 3D nanofiber scaffolds because of their low strength and low rate of cell diffusion in the thickness direction [6]. Although the microfibers have higher strength, the large fiber diameters may have a negative effect on cell adherence and proliferation [7]. A composite fiber with core/sheath structure composed of both nano- and micro-fibers is believed to be an ideal architecture, because the outer nanofibrous networks increase the surface area which is favorable for cell attachment, and the inside microfibers ensure the sufficient mechanical strength [8].

Silver nanowire is a special one-dimensional conductive nanomaterial with high aspect ratios and is capable to form an interconnected network facilely in polymer matrix [9]. In addition, there is an increasing interest toward exploitation of nano-silver technology in the development of biomaterials, aimed at taking advantage of the antibacterial property of silver [10].

In this study, electrospinning technique with two spinnerets was used to fabricate very fine PVA nanofibers and the microfibers containing AgNWs (PVA/AgNWs microfibers) was used as collector. Under the effect of electric field force, the nanofibers were deposited on the surface of the microfibers directly. The final composite fibers were composed of PVA/AgNWs microfibers as cores and PVA nanofibers as sheaths. Then the antibacterial property and cell adhesion of the micro/nano fibers were tested. 


\section{Experimental}

\section{Materials}

PVA (degree of polymerization 1750, alcoholysis degree 98\%,Mw=74 800), ethylene glycol (EG), sodium chloride $(\mathrm{NaCl})$, acetone, dimethyl sulfoxide (DMSO), methanol, silver nitrate $\left(\mathrm{AgNO}_{3}\right)$, polyvinyl pyrrolidone (PVP, Mw=130000) were purchased from Sinopharm Chemical Reagent Co., Ltd (China).

\section{Micro/Nano Composite Fibers Preparation}

AgNWs were produced by a solvothermal process according to the report of Moreno et al [11], and dispersed in a mixed solvent $\left(\mathrm{DMSO} / \mathrm{H}_{2} \mathrm{O}_{\mathrm{vol}}=3 / 1\right)$. Then a certain amount of $\mathrm{AgNW}$ s dispersion was added into 16\% PVA solution (the same solvent as the AgNWs dispersion) to prepare spinning dopes, and the PVA/AgNWs microfibers was fabricated by wet spinning using a self-made spinning apparatus [12]. A set of PVA/AgNWs microfibers with AgNWs mass fractions of 0, 0.25, 0.5, 0.75, 1 and $1.25 \mathrm{wt} \%$ to the PVA weight were obtained.

Electrospinning technique was used to prepare micro/nano composite fibers. Two positively charged spinnerets were placed opposite each other. A single prepared PVA/AgNWs microfiber was located in the middle of the spinnerets and across the electric field to act as a grounded collector in electrospinning process, then the nanofibers could deposit on the surface of the PVA/AgNWs microfibers directly. The electrospinning was conducted with the applied voltage maintained at 7.5 $\mathrm{kV}$, PVA aqueous solution concentration maintained at $7 \mathrm{wt} \%$, and the electrospinning distance was $15 \mathrm{~cm}$.

\section{Post-treatment of Micro/Nano Composite Fibers}

Prior to the antimicrobial test and cell adhesion assay, the micro/nano composite fibers and the blank (PVA nanofiber mat) undertook methanol treatment for $24 \mathrm{~h}$ and dried in a ventilated hood at room temperature for $24 \mathrm{~h}$.

\section{Cell Culture and Adhesion}

Human osteoblast MC3T3 cells were donated by Medical College of Soochow University and the cells from second passage were used for the experiments reported here. The treated samples were sterilized by exposure to UV light for $30 \mathrm{~min}$, rinsed briefly with PBS for 3 times. Then the samples were placed in a 24 -well plate and seeded with the second passage primary osteoblasts $\left(4 \times 10^{3}\right.$ cell/hole) in complete medium, consisting of DMEM-high glucose (Sigma) supplemented with $10 \%$ FBS and $1 \%$ antibiotics-antimycotics (1\% penicillin-streptomycin, Sigma). The cells were allowed to grow in a humidified atmosphere containing $5 \%$ of $\mathrm{CO}_{2}$ for $24 \mathrm{~h}$ at $37{ }^{\circ} \mathrm{C}$. After 1 day of culture, the cell-seeded samples were rinsed with PBS, fixed with $10 \%$ neutral-buffered formalin and stained with 4',6-diamidino-2-phenylindole (DAPI, Qcbio Science\& Technology Co., Ltd) to visualize the presence of live cells.

\section{Characterization}

The morphologies of PVA/AgNWs microfibers and micro/nano composite fibers were investigated over a Hitachi S-4700 scanning electron microscope (SEM) after gold coating. The antimicrobial activities of the micro/nano composite fibers were tested against Escherichia coli (ATCC 8099) and Staphylococcus aureus (ATCC 6538). Agar plates containing test samples and control (blank) were incubated at certain temperatures $\left(\right.$ E. coli: $30{ }^{\circ} \mathrm{C} ;$ S. aureus: $\left.37^{\circ} \mathrm{C}\right)$ for $24 \mathrm{~h}$. The blue-stained live cells were visualized using a GFM-500 fluorescence microscope.

\section{Results and Discussion}

Fig.1 (a) and (b) show the photographs of continuous PVA/AgNWs microfiber and micro/nano composite fiber. As seen in the SEM image of Fig. 1 (a'), the surface of the PVA/AgNWs microfiber 
containing $0.5 \mathrm{wt} \%$ AgNWs presents a relatively smooth morphology without apparent agglomeration. Fig.1 (b') is the SEM image of micro/nano composite fiber, it is clear that the nanofibers successfully cover the microfiber and form a relatively uniform sheath. Through the software of the SEM, the diameter of the obtained nanofibers can be measured in the range of $300-700 \mathrm{~nm}$.
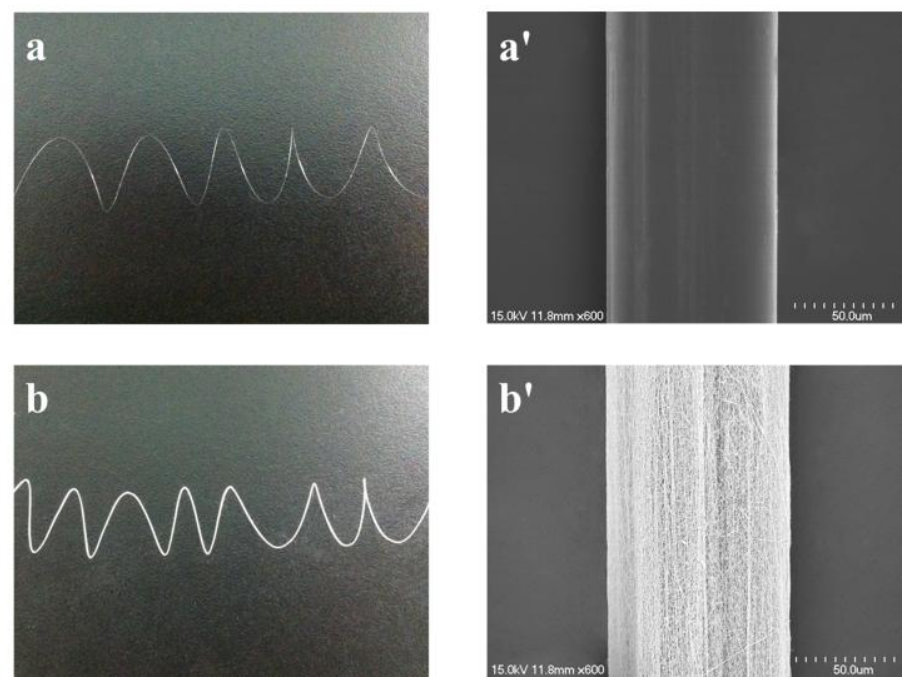

Fig.1 Photographs of (a) PVA/AgNWs microfiber and (b) micro/nano composite fiber. SEM images of (a') PVA/AgNWs microfiber and ( $b^{\prime}$ ) micro/nano composite fiber. AgNWs content is $0.5 \mathrm{wt} \%$.
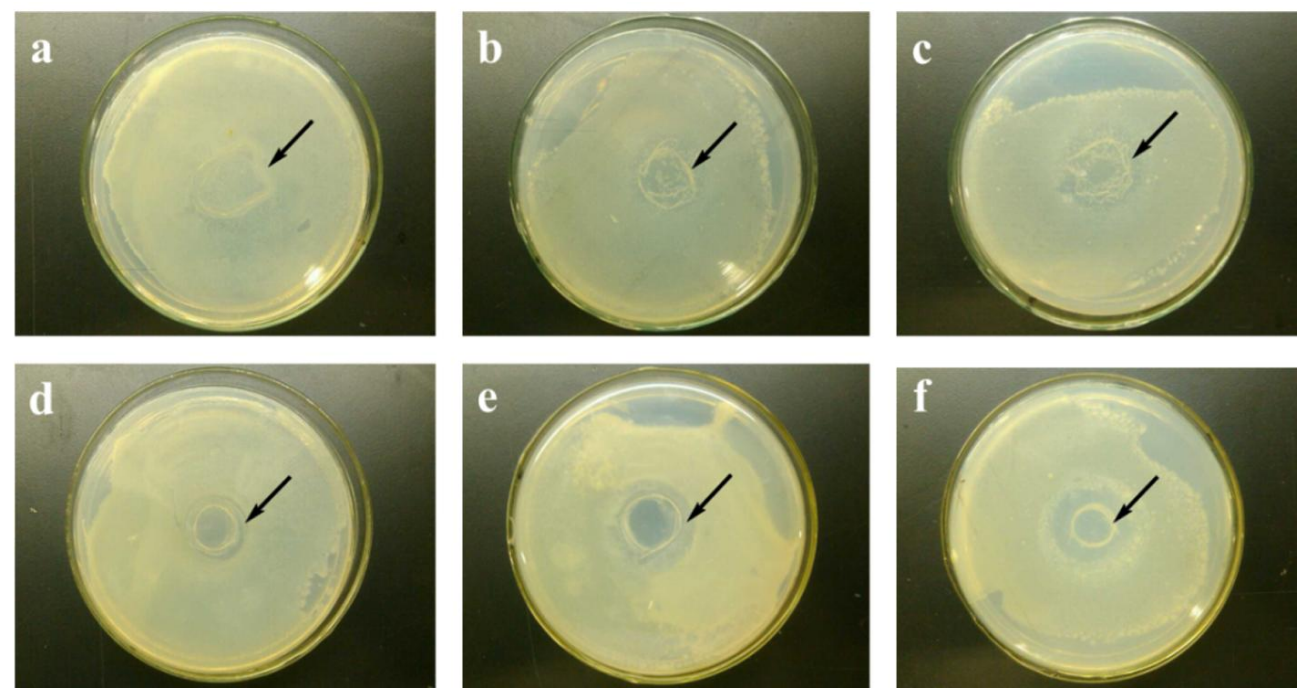

Fig.2 Test results of antimicrobial activities of micro/nano composite fibers against $E$. coli with different AgNWs contents: (a) $0 \mathrm{wt} \%$; (b) $0.25 \mathrm{wt} \%$; (c) $0.5 \mathrm{wt} \%$; (d) $0.75 \mathrm{wt} \%$; (e) $1 \mathrm{wt} \%$ and (f) 1.25 $\mathrm{wt} \%$. The arrowheads point toward the micro/nano composite fibers.

PVA nanofibrous nonwoven mat is water-soluble and will instantaneously shrink, then become a clear, gelatinous material with destroyed nano structure when immersed in water. So the samples used for antimicrobial test and cell adhesion assay were all treated by methanol to crosslink the PVA [13].

For the antimicrobial test, the composite fibers were placed in bacteria-inoculated agar plates, and then visualized for antibacterial activity after being incubated for $24 \mathrm{~h}$ at $30^{\circ} \mathrm{C}$ for $E$. coli and $37^{\circ} \mathrm{C}$ for $S$. aureus. As shown in Fig. 2(a) (c), there are no clear inhibition zones against E. coli around the micro/nano composite fibers with AgNWs content below $0.5 \mathrm{wt} \%$, while the composite fibers with over $0.75 \mathrm{wt} \%$ AgNWs exhibit noticeable antimicrobial activity as indicated by the clear inhibition zones against $E$. coli as shown in Fig. 2(e) (f). 

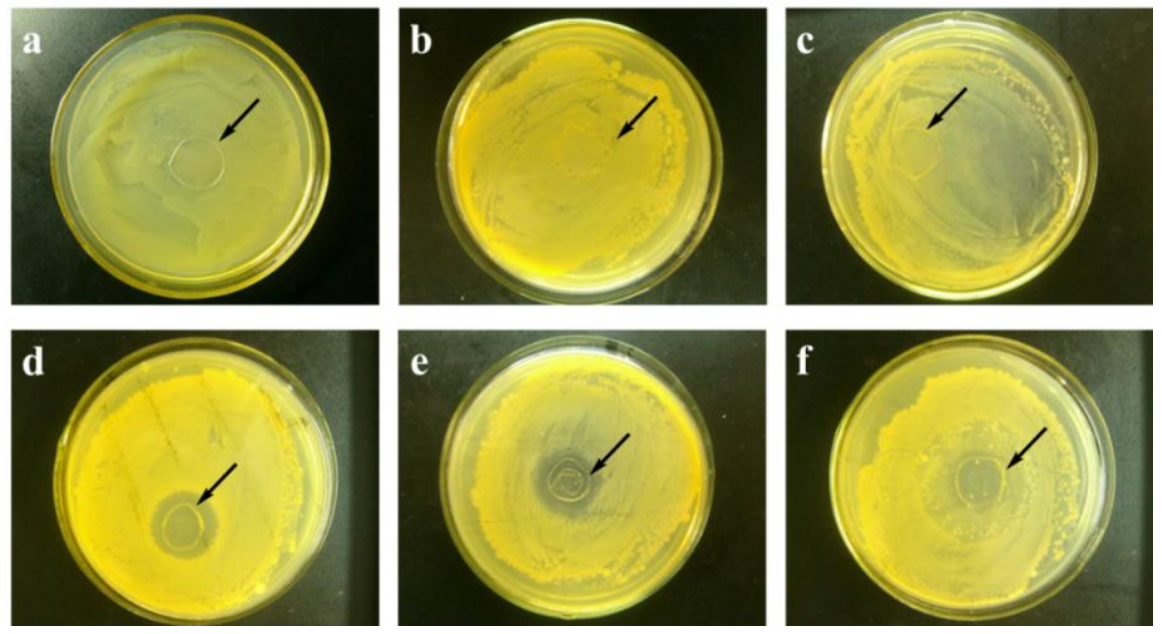

Fig.3 Test results of antimicrobial activities of micro/nano composite fibers against $S$. aureus with different AgNWs contents: (a) $0 \mathrm{wt} \%$; (b) $0.25 \mathrm{wt} \%$; (c) $0.5 \mathrm{wt} \%$; (d) $0.75 \mathrm{wt} \%$; (e) $1 \mathrm{wt} \%$ and (f) 1.25 $\mathrm{wt} \%$. The arrowheads point toward the micro/nano composite fibers.

The antimicrobial activity against $S$. aureus (see Fig. 3) is similar with E. coli. When the metallic silver is placed in water, or in the air, trace amounts of silver ions diffuse out from the free surface of the silver, which has a bactericidal effect [14]. The result indicates that although the PVA/AgNWs microfibers are wrapped by nanofibers, the micro/nano composite fibers still have a satisfactory antibacterial property, which is suggested to be caused by $\mathrm{Ag}^{+}$diffusion out from core microfiber to the surface of nanofiber sheath because of slight swelling of PVA when put the samples in agar plates.
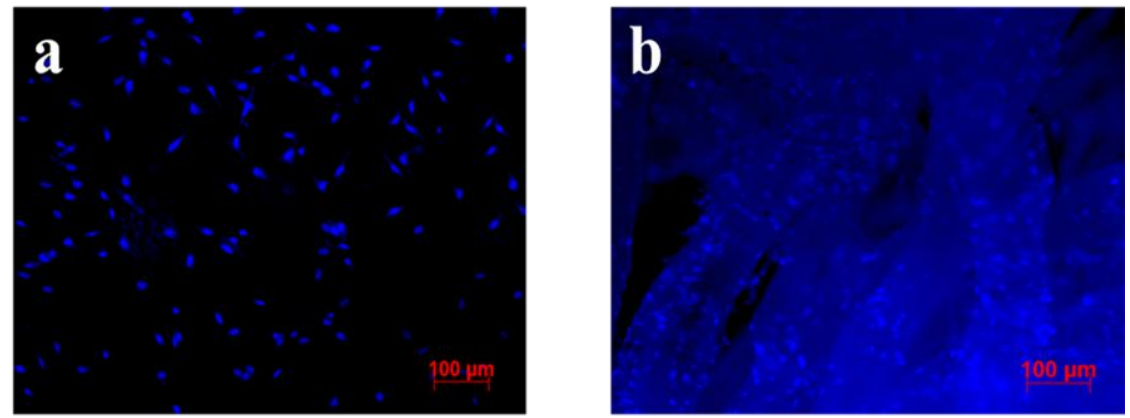

Fig.4 Fluorescence microscopy demonstrating DAPI-stained human osteoblasts growing on the (a) pure PVA nanofiber mat; (b) scaffold consisting of micro/nano composite fibers after 1 day of culture (AgNWs content of the core fibers is $1.25 \mathrm{wt} \%$ ).

The facilitation of cell adhesion on the pure PVA nanofiber mat and the scaffold made of micro/nano composite fibers was investigated by culturing human osteoblast MC3T3 cells and staining them with DAPI. Fig. 4 shows the distribution of human osteoblasts cultured on various fibers during 1 day. It is well known that the architecture of nanofiber mats with a high surface area is similar to that found in most natural extra-cellular matrices and thus promote cell adhesion, migration and proliferation [15,16]. As shown in Fig. 4, the living cells evenly spread over both the pure PVA nanofiber mat and the micro/nano composite fiber scaffold, but the cells on the composite fiber scaffold can adhere in a 3D direction.

\section{Summary}

PVA nanofibers coating on PVA/AgNWs microfibers were realized by a modified electrospinning apparatus. The antibacterial property and cell adhesion of the micro/nano fibers have been tested. It was found that the composite fibers possessed good antibacterial activity against $E$. coli and $S$. aureus 
because of the addition of AgNWs. The special micro/nano composite fibers exhibited not only adhesion of human osteoblasts in a 3D direction, but also a high density of the cells adhered to the fibers. It is suggested that the proposed technology creates the opportunity to produce micro/nano composite fibers with core/sheath morphology for a wide variety of applications such as tissue engineering, antibacterial materials and drug delivery.

\section{Acknowledgement}

This research was supported by Natural Science Fund of Jiangsu Province (BK2012623) and a Project Funded by the Priority Academic Program Development of Jiangsu Higher Education Institutions.

\section{References}

[1]G. Paradossi, F. Cavalieri, E. Chiessi, S. Chiara and M.K. Cowman: J. Mater. Sci. Mater. Med. Vol.14 (2003), p. 687

[2]H.S. Mansur and H.S. Costa: Chem. Eng. J. Vol. 137 (2008), p.72

[3]H. Yoshimoto, Y.M. Shin, H. Terai and J.P. Vacanti: Biomaterials Vol. 24 (2003), p. 2077

[4]H.S. Yoo, T.G. Kim and T.G. Park: Adv. Drug Deliver. Rev. Vol. 61 (2009), p. 1033

[5]Y. Zhou, D. Yang, X. Chen, Q. Xu, F. Lu and J. Nie: Biomacromolecules Vol. 9 (2007), p. 349

[6]G.H. Kim and W.D. Kim: J. Biomed. Mater. Res. B Vol. 81(2007), p. 104

[7]A. Thorvaldsson, H. Stenhamre, P. Gatenholm and P. Walkenström: Biomacromolecules Vol. 9 (2008), p. 1044

[8]N. Kasoju, R.R. Bhonde and U. Bora: Mater. Lett. Vol. 63 (2009), p. 2466

[9]F. Xu and Y. Zhu: Adv. Mater. Vol. 24 (2012), p. 5117

[10]W. Lu, G. Liu, S. Gao, S. Xing and J. Wang: Nanotechnology Vol. 19 (2008), 445711

[11]I. Moreno, N. Navascues, M. Arruebo, S. Irusta and J. Santamaria: Nanotechnology Vol. 24 (2013) 275603

[12]P. Zhang, D. Qiu, H. Chen, J. Sun, J. Wang, C. Qin and L. Dai: J. Mater. Chem. A Vol. 3 (2015), p. 1442

[13]L. Yao, T.W. Haas and A. Guiseppi-Elie: Chem. Mater. Vol. 15 (2003), p. 1860

[14]R. Kumar and H. Münstedt: Biomaterials Vol. 26 (2005), p. 2081

[15]K.T. Shalumon, N.S. Binulal, N. Selvamurugan, S.V. Naira, D. menona, T. Furuikeb, H. Tamurab and R. Jayakumara: Carbohydr. Polym. Vol. 77 (2009), p. 863

[16]B. Duan, X. Yuan, Y. Zhu, Y. Zhang, X. Li, Y. Zhang and K. Yao: Eur. Polym. J. Vol. 42 (2006), p. 2013 